\title{
Credit Spreads And Systematic Risk In The U.S. Banking Industry - A Neural Network Model Approach
}

Mark A. Anderson, Minnesota State University, USA

Frantz Maurer, KEDGE Business School \& University of Bordeaux, IRGO, EA4190, France

\begin{abstract}
This paper shows that systematic risk in the U.S. banking industry displayed historical responsiveness to variations in the AAA-Baa credit spread. Critically, through the development of a series of single hidden layer perceptron neural network models, the principal credit spreads in the fixed income market catalyzed a defined regime shift in systematic risk proximate the financial crisis, and was more influential to the quantification of realized systematic risk than the statistical specifications of beta. As an intriguing result of the learned model simulations, the beta slope coefficients for the largest banks in the study exhibited significant acceleration in the statistical dependence on credit spread variations.
\end{abstract}

Keywords: Banking Industry; Risk; Credit Spreads; Neural Networks; Regime Shifting

\section{INTRODUCTION}

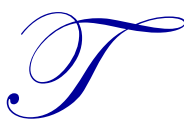
variation in risky asset prices, traditionally accepted return covariances between and across asset classes broke down. Interestingly, larger banks and in general firms with higher market capitalization levels and greater trading frequency, have traditionally experienced notably less variation on beta nonstationarity than banks with smaller market capitalization (Brenner \& Smidt, 1977; Trecroci, 2013).

The emphasis of this paper resides in the influences on and patterns of nonstationarity in systematic risk in the U.S. banking industry during the 2002 through 2011 period in aggregate. Of greater import, however, are the two critical dimensions - size and periodicity - that segment the analysis and establish pervasive evidence of a regime shift in risk drivers and dramatically elevated systematic risk levels in larger institutions vis-à-vis smaller banking companies. It is the comparison of variation between two mutually exclusive pre/proximate financial crisis subperiods (Allen \& Faff, 2012) and two sets of banks subdivided by aggregate market capitalization that exposes shifts in systematic risk that appears to exert a bias in favor of smaller market capitalization banks at the time de-risking was the theme.

Also, our study represents a notably novel incorporation of artificial neural networks (see e.g., De Veaux \& Ungar, 1997; Jones, 2004) in combinations with traditional tools to isolate greater stability in market risk for smaller banks at a time when greater stability represents the paramount issue. The analysis extends into the identification of a decided shift in the variation of systematic risk and the entry of a new temporary paradigm in which credit spreads are more influential than the formulaic construct.

The rest of this paper proceeds as follows. The neural network modeling framework is presented in Section 2. Then, Section 3 discusses empirical results. Finally, Section 4 summarizes the main findings and concludes. 


\section{THE NEURAL NETWORK-MODELING FRAMEWORK}

\section{Data and Sample}

Of the 348 banking companies listed and actively traded on the NYSE, AMEX, or NASDAQ at December 31,2011 , the 100 banks that populate our study represent $94.6 \%$ of the aggregate market capitalization for the group. This initial sample is then segmented by market capitalization into two equal-sized sample subsets, namely the 50 largest and the remaining 50 banking companies. Interestingly, the 50 banks that comprise the subset of larger banks within the study constituted $97.9 \%$ of the trading volume inherent in the sample and $96.7 \%$ of the trading volume realized by the entire group of listed banks.

The study covers the period commencing December 31, 2001 through December 31, 2011. The specification of the largest domestic U.S. banking companies is derived through access to market capitalization information and trading metrics from the NYSE, and NASDAQ ${ }^{\circledR}(2012)$. The price and return information resident in the analytical framework are extracted from the CRSP database. The prices and returns are stock split-adjusted and reflect the consideration of cash and/or stock dividends. Daily security returns are determined utilizing natural logarithms of daily prices to derive continuously compounded returns.

\section{Systematic Risk Nonstationarity}

The initial stage of the study entails the selection of an approach to establish and quantify systematic risk nonstationarity. To reduce biases potentially introduced by the consideration of a singular systematic risk derivation approach, commonly typified by the ordinary least squares (OLS) methodology, the pattern of beta nonstationarity for the sample sets is evaluated within an expanded purview of systematic risk. This expansion encompasses OLSbeta as well as Scholes-Williams (1977) beta, two specifications of Dimson's (1979) beta, namely single-day and two-day return lags and leads, and the arithmetic mean of these four intertemporal formulaic variations.

The artificial neural network dimension of the study critically entailed the incorporation of relative intertemporal systematic risk coefficients derived from daily and annual interval estimation catalyzed by return (individual security, the risk-free rate, and the market - hence, the individual and market risk premiums) intervals measured over rolling daily and annual temporal periodicity intervals. With temporal nonstationarity and the desire to synchronize the daily consideration of systematic risk and the derivation of the intertemporal time-varying (rolling) beta measured daily over one-year periods, two independent covariates have been included as possible relative systematic risk factors in the neural network models.

The first, $\beta_{i \mid D}$, represents the application of daily return intervals and daily measurement periods, where individual beta metrics premised on the application of the CAPM formulaic construct to align daily individual risk premiums with daily market risk premiums have been included as a covariate. The second, $\beta_{i \mid A}$, represents the application to annual individual risk premiums and the corresponding annual market risk premiums (i.e., an intertemporal approach conditioned on annual versus daily holding period returns).

\section{The Lagged Credit Spread Factor}

Essential to the exercise was the investigation into incremental plausible correlation and potentially causal explanatory factors ascribed to the large expansion in the volatility of systematic risk in the larger bank subset. Given the visually arresting disposition of risk in the U.S. banking industry from 2002 through 2011 - and specifically from 2007 through 2011 - the relationship between interest rates on fixed income securities of varying credit quality levels (interest rate credit spreads) and systematic risk variation has been isolated.

The selection of a specific credit spread and the alignment with a singular unique lag emanated from a variety of credit spread combinations and the analysis of approximately two hundred lag and lead days. The most promising credit relationship considered and observed was the aggregate credit spread between the U.S. Constant Maturity Treasury (CMT hereafter) with a 10-year maturity and the Baa Corporate Bond Index as published in the H.15 Selected Interest Rates (FRB H.15, 2013) release of the U.S. Federal Reserve Board of Governors (FRB). The 
FRB Baa Corporate Bond Index represents Moody's Seasoned Baa Corporate Bond Yield for bonds with remaining maturities as close as possible to 30 years.

The consideration of a range of lag periods ultimately resulted in a specified lag period derived through the synchronization of the cyclical peak intertemporal mean beta slope coefficient $\left(\beta_{\mathrm{OLS}}\right)$ and the peak CMT-Baa spread, denoted as $C S_{L}$. The impact of the extension of the calibration to accommodate the 406-day lag in credit spreads to align with variations in the mean beta coefficients on the correlation coefficients is summarized in Table 1. This table reflects the variations and volatility in the correlation coefficients over ten annual observation periods ended December 31, 2011.

Table 1. Temporal Correlation Coefficients $-C S_{L}$ and $\beta_{\mathrm{OLS}}$

\begin{tabular}{lllrrrrrrrr}
\hline & $\boldsymbol{\rho}_{\mathbf{1 0}}$ & $\boldsymbol{\rho}_{\mathbf{9}}$ & $\boldsymbol{\rho}_{\mathbf{8}}$ & $\boldsymbol{\rho}_{\mathbf{7}}$ & $\boldsymbol{\rho}_{\mathbf{6}}$ & $\boldsymbol{\rho}_{\mathbf{5}}$ & $\boldsymbol{\rho}_{\mathbf{4}}$ & $\boldsymbol{\rho}_{\mathbf{3}}$ & $\boldsymbol{\rho}_{\mathbf{2}}$ & $\boldsymbol{\rho}_{\mathbf{1}}$ \\
\hline $\boldsymbol{\beta}_{\mathbf{F}} \rightarrow \boldsymbol{C} \boldsymbol{S}_{\boldsymbol{L}}$ & 0.116 & 0.195 & 0.430 & 0.658 & 0.669 & 0.635 & 0.565 & 0.740 & 0.695 & $(0.038)$ \\
$\boldsymbol{\beta}_{\mathbf{L}} \rightarrow \boldsymbol{C} \boldsymbol{S}_{\boldsymbol{L}}$ & 0.333 & 0.416 & 0.567 & 0.738 & 0.756 & 0.725 & 0.645 & 0.760 & 0.752 & 0.254 \\
$\boldsymbol{\beta}_{\mathbf{S}} \rightarrow \boldsymbol{C} \boldsymbol{S}_{\boldsymbol{L}}$ & $(0.092)$ & $(0.061)$ & 0.125 & 0.307 & 0.301 & 0.372 & 0.440 & 0.695 & 0.611 & $(0.397)$ \\
\hline
\end{tabular}

Note: $C S_{L}$ denotes the daily credit spread between the U.S. 10-year CMT and the Baa Corporate Bond Index with a lag of 406 calendar days. $\beta_{\text {OLS }}$ denotes the intertemporal mean beta slope coefficient derived through ordinary least square. $\beta_{\mathrm{F}}, \beta_{\mathrm{L}}$, and $\beta_{\mathrm{S}}$ denote the beta slope coefficients estimated over (i) the full sample $S_{F}$ of 100 publicly traded banking companies, (ii) the first subsample $S_{L}$ of the 50 largest banks ranked by market capitalization, and (iii) the second subsample $\mathrm{S}_{\mathrm{S}}$ with the remaining 50 banks, respectively. Figures displayed into brackets indicate negative correlations. The notation $\rho_{i}, i=10, . ., 1$ reflects the number of years that end with the 2011 observation included in the correlation. $\rho_{10}$ is the correlation coefficient between the paired variables for the ten year period 2002 through $2011 ; \rho_{9}$ is the correlation for the nine year period 2003 through 2011 , etc.

Multiple conclusions extend form Table 1, but importantly, correlations coefficients peak for both bank sets when observed for the 2009 through 2011 period, but exhibit elevation for $S_{L}$ for seven of the ten observation periods. While escalation in correlation coefficients for both bank sets and $\mathrm{S}_{\mathrm{F}}$ occur when observed for the 2009 through 2001 calendar years, correlation coefficients for the two subsets are observably higher than for $\mathrm{S}_{\mathrm{F}}$, indicative of differing relationships. Additionally, correlation coefficients reveal periods of negative correlation, particularly when observed for $\mathrm{S}_{\mathrm{S}}$. Table 2 reveals the correlation coefficients specified over shorter intervals defined by the ten calendar-years observed in the analysis. This table isolates a generally wide variation in correlation between the two variables viewed by set (subset) or by observation period, with the coefficients specified for 2009, and to a modestly lesser extent 2010, revealing conspicuously strong correlation biases.

Table 2. Annual Correlation Coefficients $-C S_{L}$ and $\beta_{\mathrm{OLS}}$

\begin{tabular}{lllllllllll}
\hline & $\boldsymbol{\rho}_{\mathbf{2 0 0 2}}$ & $\boldsymbol{\rho}_{\mathbf{2 0 0 3}}$ & $\boldsymbol{\rho}_{\mathbf{2 0 0 4}}$ & $\boldsymbol{\rho}_{\mathbf{2 0 0 5}}$ & $\boldsymbol{\rho}_{\mathbf{2 0 0 6}}$ & $\boldsymbol{\rho}_{\mathbf{2 0 0 7}}$ & $\boldsymbol{\rho}_{\mathbf{2 0 0 8}}$ & $\boldsymbol{\rho}_{\mathbf{2 0 0 9}}$ & $\boldsymbol{\rho}_{\mathbf{2 0 1 0}}$ & $\boldsymbol{\rho}_{\mathbf{2 0 1 1}}$ \\
\hline $\boldsymbol{\beta}_{\mathbf{F}} \rightarrow \boldsymbol{C} \boldsymbol{S}_{\boldsymbol{L}}$ & 0.724 & 0.758 & $(0.924)$ & $(0.584)$ & $(0.394)$ & 0.149 & $(0.378)$ & 0.968 & 0.744 & $(0.038)$ \\
$\boldsymbol{\beta}_{\mathbf{L}} \rightarrow \boldsymbol{C} \boldsymbol{S}_{\boldsymbol{L}}$ & 0.729 & 0.023 & $(0.950)$ & $(0.637)$ & $(0.233)$ & $(0.403)$ & $(0.069)$ & 0.967 & 0.768 & 0.254 \\
$\boldsymbol{\beta}_{\mathbf{S}} \rightarrow \boldsymbol{C} \boldsymbol{S}_{\boldsymbol{L}}$ & 0.617 & 0.931 & $(0.910)$ & $(0.530)$ & $(0.514)$ & 0.475 & $(0.614)$ & 0.967 & 0.708 & $(0.397)$ \\
\hline
\end{tabular}

\section{Neural Network Model Calibration}

The observations and beta coefficients follow three temporal constructs: two five-year windows, denoted as $\mid \mathrm{F} 5$ and ${ }_{\mid \mathrm{S} 5}$, and referring to the pre-financial crisis period (2002-2006) and the proximate financial crisis period (2007-2011) respectively, and the aggregate ten-year period (2002-2011) denoted as ${ }_{110}$. For the observations arising out of three time-periods for each of three sets or subsets of banks $\left(\mathrm{S}_{\mathrm{F}}, \mathrm{S}_{\mathrm{L}}\right.$, and $\left.\mathrm{S}_{\mathrm{S}}\right)$, nine scenarios frame for the neural network independent variable importance analysis in the pursuit of explanations to the large relative acceleration in variability of systematic risk in the large bank subset and the presumed regime shift in variable influence.

Endemic to each of the nine permutations, $C S_{L}, \beta_{i \mid D}$ and $\beta_{i \mid A}$ comprise the three unique covariates, and $\mathrm{M}_{\mathrm{DIR}}$ and $V_{\text {DIR }}$ reflect the independent directional factors. The isolation of the direction of volatility (variance relative to market variance) and the direction of market returns represent plausible factor contributors as $\mathrm{M}_{\mathrm{DIR}}$ and $\mathrm{V}_{\mathrm{DIR}}$. With each set of five independent drivers regressed against the corresponding mean intertemporal $\beta_{\text {OLS }}, 50$ neural network models simulated an aggregate of 450 learned models. The learning models were calibrated to draw from the 2,519 observations premised on a $5 \%$ level of test significance and a targeted maximum statistical margin of error $\left(M E_{t}\right.$ 
hereafter) of $2.5 \%$ with the training sequence set to $70 \%$ of the observations and a test sequence applied to the remaining $30 \%$ of the observations ${ }^{1}$.

To put it differently, each of 450 unique artificial neural network models extended from the creation of the best-fit nonlinear learned models derived via the analysis of approximately 1,763 of 2,519 (or 881 and 882 for |F5 $_{\text {F }}$ and $\mid \mathrm{s} 5$ respectively) combinations of the six variables. Of the 45 factors and covariates (independent drivers) that constituted the 450 neural network models, there were four instances in which the actual margin of error $\left(m e_{a c t}\right)$ exceeded the target $M E_{t}$ and none were in excess of $2.8 \%$. To establish the number of iterations considered to establish the mean independent variable importance, as noted, an iterative process was developed for each of the nine combinations of set/subset composition and temporality that extended from the sample followed the guideline formula $n=\left(z_{\alpha}^{2} * \sigma_{v i}^{2}\right) / M E_{t}^{2}$ (DeFusco et al., 2007).

In this target sample size guideline, $z$ denotes the critical test value at a level of test significance equal to $\alpha$, $\sigma_{v i}{ }^{2}$ reflects the variance of the respective independent variable importance measures (and $\sigma_{v i}$ defies the standard deviation), and $M E_{t}$ specifies the target margin of error in the estimation process. Thus, $n$ is a function of $\sigma_{v i}$, and $\sigma_{v i}$ arises out of the variations realized in the independent variable importance statistics in $n$ simulations of the model. Target and actual sample sizes utilized $\alpha=5 \%$ and a $2.5 \%$ target margin of error. The actual margin of error, $m e_{a c t}$, extended from the target formulaic construct modified as $m e_{a c t}=\left(z_{\alpha^{*}} \sigma_{v i}\right) / \sqrt{n}$.

\section{Relative Contribution of Predictor Factors and Covariates}

The inclusion of the neural network-modeling framework represents a dynamic supplement to alternative analytical techniques and offers the power to measure the relative contribution of the noted variables within the construct of independent variables and covariates regressed against a singular dependent variable, set as the most accurate realized intertemporal systematic risk metric, $\beta_{\text {OLS }}$, in this analysis. Thus, as a source of learned dependent variable behavior, the measures of independent variable importance for each and all of the specified neural network models proffer a viable and valid comparative tool in the consideration of which, if any, of the cited independent variables (and/or covariates) contribute disproportionately meaningful explanatory influence in the learned dynamic model.

For purposes of analytical evaluation, the study initially considered six unique and reflexive neural network models. The singular neural network model design predicated on a complement of five factors or covariates set within three temporal ranges and three constitutional sets coalesced into nine unique scenarios that catalyze 45 sets of independent variable importance statistics. This aggregation of 45 sets of independent variable importance statistics arise from the 50 neural network simulations processed for each of the nine set and periodicity combinations, reflective of individual variable importance statistics ${ }^{2}$.

The investigation into factors correlated to the regime shift and the reversal in systematic risk variability between the two sets of banks incorporated Multilayer Perceptron neural network architecture with preference isolated to the single hidden layer activation variation. The optimization algorithm followed the scaled conjugate gradient technique. In this manner, the historical relationship between the factors and covariates and the dependent variable (the actual realized beta coefficient) engaged more complex nonlinear explanations of fit than feasible via traditional linear formulaic constructs and common nonlinear estimation approaches. Created in the training mode as a learned model and generated in the testing stage, the aggregation of 50 neural network simulations for each of the nine combinations of sample set/subset and temporal specification reflect statistical significance and offer explanations to the changed behavior patterns observed in systematic risk variation.

\footnotetext{
${ }^{1}$ In aggregate, the sample for the 2002 through 2011 period yielded 2,519 temporal observations The pre/proximate financial crisis two subperiods (2007-2011/2002-2006) include 1,259 and 1,260 observations, respectively.

${ }^{2} 2,250=9$ unique scenarios $\times 5$ factors or covariates $\times 50$ neural network simulations.
} 


\section{EMPIRICAL RESULTS AND DISCUSSION}

\section{Absolute Deviation in Systematic Risk Estimates}

As noted, five systematic risk derivation approaches are used. They are reflective of risk estimates derived through ordinary least squares $\left(\beta_{\mathrm{OLS}}\right)$, the Dimson (1979) technique with one and two-day lags $\left(\beta_{\mathrm{D} 1}\right.$ and $\left.\beta_{\mathrm{D} 2}\right)$, the Scholes and Williams (1977) methodology $\left(\beta_{\mathrm{sw}}\right)$, and the arithmetic mean of the four constructs $\left(\beta_{\mu}\right)$. An analysis of realized beta slope coefficients within the context of (rolling) intertemporal daily return intervals applied to riskspecify the respective daily market risk premiums as the independent variable regressed against the corresponding daily individual risk premiums reveals a state of general superiority in the accuracy of $\beta_{\mathrm{OLS}}$ versus $\beta_{\mathrm{D} 1}, \beta_{\mathrm{D} 2}, \beta_{\mathrm{SW}}$, and the mean of the four variations, $\beta_{\mu}$.

The results of the analysis of the absolute deviation in beta slope coefficients across the five varied approaches to the measurement of systematic risk affirms the isolation of $\beta_{\mathrm{OLS}}$ as the most accurate of the techniques considered within the study decision criteria. Analytical results also endorse the selection of the OLS-beta as the systematic risk factor variables included in the analysis of variable importance within the set of summary results generated by the neural network model simulations. In each of the three observation intervals - the first five years, the second five years, and the full ten-year period - the specification of risk via $\beta_{\text {OLS }}$ resulted in the most accurate realized beta slope coefficients. Results are presented in Table 3.

Table 3. Absolute Beta Coefficient Relevance - Viewed Temporally

\begin{tabular}{lccccc}
\hline & $\boldsymbol{\beta}_{\text {OLS }}$ & $\boldsymbol{\beta}_{\mathbf{D} 1}$ & $\boldsymbol{\beta}_{\mathbf{D} 2}$ & $\boldsymbol{\beta}_{\mathbf{S W}}$ & $\boldsymbol{\beta}_{\boldsymbol{\mu}}$ \\
\hline$\left|\boldsymbol{\beta}_{\mathbf{F} \mid \mathbf{1 0}}-\boldsymbol{\beta}_{\boldsymbol{m}}\right|$ & 0.046 & 0.084 & 0.094 & 0.085 & 0.077 \\
$\left|\boldsymbol{\beta}_{\mathbf{F} \mid \mathbf{F 5}}-\boldsymbol{\beta}_{\boldsymbol{m}}\right|$ & 0.074 & 0.184 & 0.185 & 0.183 & 0.156 \\
$\left|\boldsymbol{\beta}_{\mathbf{F} \mid \mathbf{5 5}}-\boldsymbol{\beta}_{\boldsymbol{m}}\right|$ & 0.042 & 0.073 & 0.113 & 0.074 & 0.076 \\
$\left|\boldsymbol{\beta}_{\mathbf{F | F 5} \rightarrow \mathbf{1 0}}-\boldsymbol{\beta}_{\boldsymbol{m}}\right|$ & $\mathbf{0 . 0 5 4}$ & $\mathbf{0 . 1 1 4}$ & $\mathbf{0 . 1 3 1}$ & $\mathbf{0 . 1 1 4}$ & $\mathbf{0 . 1 0 3}$ \\
\hline
\end{tabular}

Note: $\left|\beta_{\text {F|TEMPORAlity }}-\beta_{m}\right|$ denotes the absolute deviation in the intertemporal beta slope coefficients across the five varied approaches used to measure the systematic risk (columns $\beta_{\mathrm{OLS}}$ to $\beta_{\mathrm{H}}$ ), where $\mathrm{F}_{\mathrm{F}}$ corresponds to the full sample of 100 banks, and ${ }_{\mid 10}$, |F5, and ${ }_{\mid \mathrm{S} 5}$ represent (i) the aggregate 10-year time period (2002-2011), (ii) the 5-year pre-financial crisis first sub-period (2002-2006), and (iii) the 5-year proximate-financial crisis second sub-period (2007-2011), respectively. The deviation is derived from the mean of the data set $\left(\beta_{m}\right)$. $\left|\beta_{\mathrm{F} \mid \mathrm{F} 5 \rightarrow 10}-\beta_{m}\right|$ is the average of the three absolute deviations calculated for each measure of beta (columns $\beta_{\mathrm{OLS}}$ to $\beta_{n}$ ).

With the evaluation context of systematic risk specification extended from the temporal dimension to the comparison at the sample subset level, the assessment of relative and absolute beta slope formulaic relevance again established the desirability of beta via ordinary least squares in each of the two subsets and in the full sample set of banks. Results are displayed in Table 4.

Table 4: Absolute Beta Coefficient Relevance - Viewed By Bank Size

\begin{tabular}{llllll}
\hline & $\boldsymbol{\beta}_{\text {OLS }}$ & $\boldsymbol{\beta}_{\mathrm{D} 1}$ & $\boldsymbol{\beta}_{\mathbf{D} 2}$ & $\boldsymbol{\beta}_{\mathbf{S W}}$ & $\boldsymbol{\beta}_{\boldsymbol{\mu}}$ \\
\hline$\left|\boldsymbol{\beta}_{\mathbf{F} \mid \mathbf{1 0}}-\boldsymbol{\beta}_{\boldsymbol{m}}\right|$ & 0.054 & 0.111 & 0.124 & 0.111 & 0.100 \\
$\left|\boldsymbol{\beta}_{\mathbf{L} \mid \mathbf{1 0}}-\boldsymbol{\beta}_{\boldsymbol{m}}\right|$ & 0.052 & 0.168 & 0.196 & 0.167 & 0.146 \\
$\left|\boldsymbol{\beta}_{\mathbf{S} \mid \mathbf{1 0}}-\boldsymbol{\beta}_{\boldsymbol{m}}\right|$ & 0.055 & 0.062 & 0.072 & 0.064 & 0.063 \\
\hline
\end{tabular}

Note: $\left|\beta_{\text {Composition } \mid 10}-\beta_{m}\right|$ denotes the absolute deviation in the intertemporal beta slope coefficients across the five varied approaches used to measure the systematic risk (columns $\beta_{\mathrm{OLS}}$ to $\beta_{\mathrm{m}}$ ). The deviation is derived from the mean of the data set $\left(\beta_{m}\right)$. F, L, and $\mathrm{S}$ correspond to (i) the full sample of 100 banks, (ii) the subsample of the 50 largest banks, and (iii) the subsample with the remaining 50 banks, respectively. $\mid 10$ indicates the aggregate 10-year time period (2002-2011).

\section{Credit Spreads and Systematic Risk - A Neural Network Explanation}

As previously mentioned, the most promising results emanate from the daily credit spread between the U.S. 10-year CMT and the Baa Corporate Bond Index with a lag of 406 calendar days (58 weeks or 279 trading days) denoted as $C S_{L}$. This relationship appears indicative of the variation in hypothetical longer-term (credit) risk-free interest rates and the corresponding low investment grade interest rate and a possibly more plausible specification of actual credit risk in an economic context. 
The development and application of a multifactor neural network model to the perplexing behavior of systematic risk during the study period reveal instructive results. The lagged credit spread (CMT-Baa or $\left.C S_{L}\right)$ was more influential in the explanation of the realized levels of systematic risk and on the time-variations in systematic risk over the study period than a combination of variables that extend from the CAPM formulaic representation of the beta slope coefficient.

The derivation of the nine sets of neural network models extended from the consideration of three covariates and two integral factors. The factors were isolated to assess the relative contribution to the variation in the realized beta coefficients attributed to directionality. The isolation of the direction of volatility (variance relative to market variance, denoted as $\mathrm{V}_{\mathrm{DIR}}$ ) and the direction of market returns (denoted as $\mathrm{M}_{\mathrm{DIR}}$ ) represented plausible factor contributors. In addition to the lagged credit spread $\left(C S_{L}\right), \beta_{\lambda \mid \mathrm{D}}$ and $\beta_{\lambda \mid \mathrm{A}}$ were incorporated into the learning models to consider the potential impact of a direct CAPM formulaic influence specified on both daily and annual return intervals to mitigate potential beta instability due to interval selection. In the notation of $\beta_{\lambda \mid \mathrm{D}}$ and $\beta_{\lambda \mid \mathrm{A}}, \beta_{\lambda}$ denotes the specific realized beta for the unique combination of temporality $\left({ }_{\mid F 5},{ }_{\mid S 5}\right.$, and $\left.{ }_{110}\right)$ and composition $\left(\mathrm{S}_{\mathrm{L}}, \mathrm{S}_{\mathrm{S}}\right.$, and $\left.\mathrm{S}_{\mathrm{F}}\right)$. The results are presented in Table 5 .

Table 5. Neural Network Model Results and Regime Switching

\begin{tabular}{|c|c|c|c|c|c|c|}
\hline & $\mathbf{V}_{\text {DIR }}$ & $\mathbf{M}_{\text {DIR }}$ & $C S_{L}$ & $\boldsymbol{\beta}_{\lambda \mid \mathbf{D}}$ & $\overline{\beta_{\lambda \mid \mathbf{A}}}$ & $\overline{\Sigma \beta_{\lambda \mid \mathbf{A}+\mathbf{D}}}$ \\
\hline SET $_{|\mathbf{F}| \mathbf{F} 5}$ & 0.017 & 0.049 & 0.480 & 0.217 & 0.237 & 0.454 \\
\hline $\mathbf{S E T}_{|\mathbf{L}| \mathbf{F} 5}$ & 0.015 & 0.042 & 0.465 & 0.181 & 0.297 & 0.478 \\
\hline SET $_{|\mathbf{S}| \mathbf{F} 5}$ & 0.016 & 0.054 & 0.454 & 0.261 & 0.215 & 0.475 \\
\hline $\mathbf{S E T}_{|\mathbf{F}| \mathbf{S 5}}$ & 0.033 & 0.038 & 0.521 & 0.201 & 0.207 & 0.408 \\
\hline $\mathbf{S E T}_{|\mathbf{L}| \mathbf{S 5}}$ & 0.034 & 0.050 & 0.518 & 0.161 & 0.237 & 0.398 \\
\hline $\mathbf{S E T}_{|\mathbf{S}| \mathbf{S 5}}$ & 0.040 & 0.044 & 0.436 & 0.199 & 0.281 & 0.480 \\
\hline $\mathbf{S E T}_{|\mathbf{F}| 10}$ & 0.019 & 0.049 & 0.416 & 0.270 & 0.246 & 0.517 \\
\hline $\mathbf{S E T}_{|\mathbf{L}| \mathbf{1 0}}$ & 0.024 & 0.049 & 0.422 & 0.242 & 0.263 & 0.505 \\
\hline $\mathbf{S E T}_{|\mathbf{S}| 10}$ & 0.019 & 0.072 & 0.352 & 0.278 & 0.279 & 0.557 \\
\hline
\end{tabular}

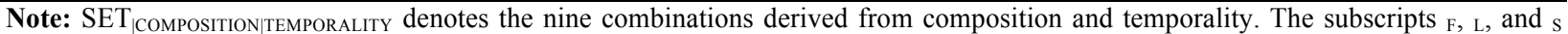
associated with ${ }_{\text {COMPosition }}$ define (i) the full sample of 100 banks, (ii) the first subsample $\mathrm{S}_{\mathrm{L}}$ of the 50 largest banks ranked by market capitalization, and (iii) the second subsample $\mathrm{S}_{\mathrm{S}}$ with the remaining 50 banks, respectively. The subscripts ${ }_{\mathrm{F} 5}$, 55 , and ${ }_{10}$ associated with |TEMPORAlity define (i) the aggregate time period (2002-2011), (ii) the pre-financial crisis first sub-period (2002-2006), and (iii) the proximate-financial crisis second sub-period (2007-2011), respectively. $\Sigma \beta_{\lambda \mid \mathrm{A}+\mathrm{D}}$ defines the combination of an intertemporal annual and daily beta slope formulas $\left({ }_{\mid A+D}\right)$, with $\beta \lambda$ denoting the specific realized beta for the unique combination of ${ }_{\mid \text {COMPOSITION }}$ and ${ }_{\mid \text {TEMPORALITY, }}$ where the nominal coefficient values $\beta_{\lambda \mid \mathrm{A}}+\beta_{\lambda \mid \mathrm{A}}=\Sigma \beta_{\lambda \mid \mathrm{A}+\mathrm{B}}$.

Four main observations and conclusions directly extend from the summarized results of 2,250 independent variable importance statistics generated through 450 learned neural network simulations for nine unique combinations of time and composition. First, the importance of the lagged (406 days) CMT-Baa credit spread $\left(C S_{L}\right.$ in Table 5) is evident across all combinations of temporality and set composition. Second, the lagged credit spread represented a more important independent variable than the variables that underlie the dependent variable in three of nine sets of simulation results $\left(C S_{L}>\Sigma \beta_{\lambda \mid \mathrm{A}+\mathrm{D}}\right.$ in Table 5). Third, the two sets of simulations that yielded the largest nominal indication of lagged credit spread importance were in the five-year period proximate the Financial Crisis for the large bank subset and the aggregate set of 100 banks. Last, as highlighted in Table 6, the importance of the lagged credit spread for the 50 large bank subset increased notably from the initial five-year period to the period proximate the Financial Crisis.

Table 6. Independent Variable Importance - Temporal Shifts

\begin{tabular}{|c|c|c|c|c|c|c|}
\hline & $\mathbf{V}_{\text {DIR }}$ & $\mathbf{M}_{\text {DIR }}$ & $C S_{L}$ & $\boldsymbol{\beta}_{\lambda \mid \mathbf{D}}$ & $\boldsymbol{\beta}_{\lambda \mid \mathbf{A}}$ & $\Sigma \boldsymbol{\beta}_{\lambda \mid \mathbf{A}+\mathbf{D}}$ \\
\hline$\overline{S_{F \mid F 5 \rightarrow S 5}}$ & 0.017 & $(0.011)$ & 0.041 & $(0.016)$ & $(0.031)$ & $(0.047)$ \\
\hline $\mathbf{S}_{\mathbf{L} \mid \mathbf{F} 5 \rightarrow \mathbf{S 5}}$ & 0.019 & 0.008 & 0.053 & $(0.020)$ & $(0.060)$ & $(0.080)$ \\
\hline $\mathbf{S}_{\mathbf{S} \mid \mathbf{F} 5 \rightarrow \mathbf{S 5}}$ & 0.024 & $(0.010)$ & $(0.018)$ & $(0.061)$ & 0.066 & 0.005 \\
\hline
\end{tabular}

Note: $\mathrm{S}_{\mathrm{F}}, \mathrm{S}_{\mathrm{L}}$, and $\mathrm{S}_{\mathrm{S}}$ denote (i) the full sample of 100 banks, (ii) the first subsample of the 50 largest banks ranked by market capitalization, and (iii) the second subsample with the remaining 50 banks, respectively. |F5 $\rightarrow$ S5 indicates the shift from the initial precrisis 5-year sub-period (2002-2006) to the proximate-financial crisis 5-year sub-period (2007-2011). $\Sigma \beta_{\lambda \mid A+D}$ defines a combination of the intertemporal daily and annual beta slope formulas $(\mid \mathrm{A}+\mathrm{D})$, with $\beta$, denoting the specific realized beta for the unique combination of |COMPOSITION and ${ }_{\mid T E M P O R A L I T Y}$, where the nominal coefficient values $\beta_{\lambda \mid \mathrm{A}}+\beta_{\lambda \mid \mathrm{A}}=\Sigma \beta_{\lambda \mid \mathrm{A}+\mathrm{B}}$. 
An apparent regime shift is evident with a principal impact on large banks $\left(\mathrm{S}_{\mid \mathrm{L}}\right)$ incident with the Financial Crisis where the variation in the influence of the conditioning state variable defined by the lagged CMT-Baa credit spread realized a significant expansion in independent variable importance. The acceleration in the importance of the independent variables reflected in Figure 1, taken in conjunction with Table 6 and the distinct variance in beta coefficients, frames a compelling case for a decided migratory pattern in systematic risk away from a more traditional and unconditional linear explanation and toward the isolation of a paradigm shift dependent on a credit spread state variable in a new risk regime.

Figure 1. Variations in Lagged Credit Spread Importance via Neural Network

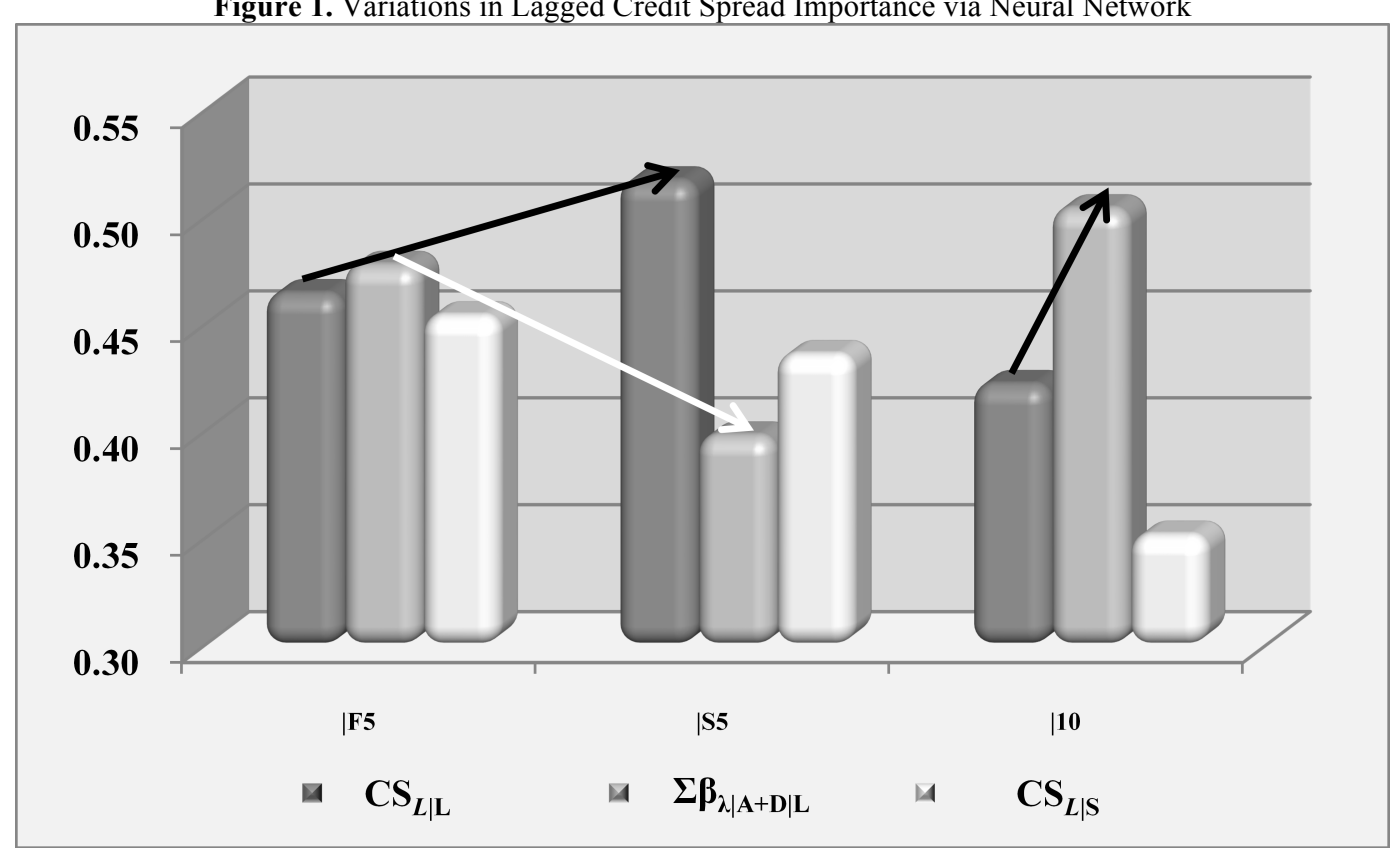

Note. ${ }_{\mid \mathrm{F} 5},{ }_{\mid \mathrm{S} 5}$, and ${ }_{110}$ define (i) the 5-year pre-financial crisis first sub-period (2002-2006), (ii) the 5-year proximate-financial crisis subperiod (2007-2011), and (iii) the 10-year aggregate time period (2002-2011), respectively. CS|L and $\mathrm{CS}_{\mid \mathrm{S}}$ denote the lagged (CMT-Baa) credit spread for the 50 largest banks subsample (|L) and the subsample with the remaining 50 banks (|S). $\Sigma \beta_{|| A+D \mid L}$ denotes a combination of an intertemporal annual and daily beta slope formula $\left({ }_{(A+D}\right)$ applied to the 50 largest banks subsample $(\mid \mathrm{L})$, with $\beta_{k}$ denoting the specific realized beta for the unique combination of $\left.\right|_{\text {|COMPOSITION }}$ and |TEMPORALITY. $_{\text {. }}$

The lagged (CMT-Baa) credit spread independent variable, in fact, was more relevant as an explanatory factor in the neural network simulations than the combination of an intertemporal (daily) slope formula and the intertemporal (annual) slope formula - both dependent on individual equity security risk premiums and market risk premiums. This anomaly surfaced a total three times in nine sets of simulations, and appears quite significant. The inference from the simulations arising from neural network analysis is that the lagged credit spread was a decidedly more influential factor in the determination of the realized levels of systematic risk than the aggregation of the formula for the slope coefficient computed for daily intervals and annual intervals.

To the extent that a journey of systematic risk refinement in the banking and other industries travels through the introduction of an exogenous state variable, such as the lagged credit spread introduced in this investigation or any other credit spread, the prospect of a paradigmatic regime shift is similarly mandated. Overall, these results catalyze the development of an artificial neural network to diagnose variations in the explanatory predictors to systematic risk nonstationarity.

\section{Potential Application in the Banking Industry}

Organizations exist to optimize long-term shareholder value - no different for publicly traded banks - and accordingly, value realization is a very direct function of real risk-adjusted or risk-specified returns. Variability of systematic risk and absolute escalation in systematic risk contribute to elevated costs of capital (Guidolin et al., 
2012). Higher beta coefficients correspond with higher costs of capital and higher required rates of return, which in turn, catalyze downward pressure on stock price appreciation or reductions in value and potentially adverse incremental earnings impacts. Our study identifies a number of critical findings that may serve to guide the approach to market risk taken by publicly traded banks and other firms to the extent the findings extend across industries. Executive teams possess the power to engage the content of the investigation to take the analysis an additional step forward to identify the characteristics of the bank set that realized less volatility in systematic risk. Further analyses of the balance sheet characteristics of large banks versus small banks and variations in asset and liability composition and relative capital levels may further contribute to the practical implementation of the findings.

\section{CONCLUSION}

This article highlights two key findings arising from artificial neural network simulations: (1) the 406-day lagged CMT-Baa credit spreads represented more important factors to intertemporal beta variation proximate the Financial Crisis for large banks (and for the full sample set of banks) than for smaller banks, and evident prior to the Financial Crisis, and (2) the critical importance exerted by lagged credit spreads on smaller banks diminished during the period proximate the Financial Crisis. Our study also supports the contention that unconditional models may be inferior to conditional models, and conditional asset-pricing models that lack a regime switching isolation and incorporation mechanism may similarly yield inferior estimates.

\section{AUTHOR INFORMATION}

Mark A. Anderson, CFA, DBA is President and CEO of BlackRidgeBANK and BlackRidge Financial, Inc., Fargo, ND, Adjunct Professor of Finance at Minnesota State University - Moorhead, MN and affiliated with Grenoble École de Management, France.

Frantz Maurer (corresponding author) is Professor of Finance at KEDGE Business School. He holds a Ph.D. in Management Science and a post-doctoral degree from the University of Bordeaux. His current research interests focus primarily on risk management in the banking industry. KEDGE Business School, 680 cours de la Libération, 33405 Talence cedex (France). Telephone: +33(0) 556845 573. E-mail: frantz.maurer@kedgebs.com

\section{REFERENCES}

Allen, D., \& Faff, R. (2012). The Global Financial Crisis: some attributes and responses. Accounting \& Finance, $52(1), 1-7$.

Brenner, M., \& Smidt, S. (1977). A simple model of non-stationarity of systematic risk. Journal of Finance, 32(4), 1081-1092.

De Veaux, R. D., \& Ungar, L. H. (1997). A brief introduction to neural networks. Technical Report, Williams College, Williamstown, MA.

DeFusco, R. A., McLeavey, D. W., Pinto, J. E., \& Runkle, D. E. (2007). Quantitative Investment Analysis. Hoboken, NJ, USA: John Wiley \& Sons, Inc.

Dimson, E. (1979). Risk measurement when shares are subject to infrequent trading. Journal of Financial Economics, 7(2), 197-226.

Guidolin, M., McMillan, D. G., \& Wohar, M. E. (2012). Time varying stock return predictability: Evidence from US sectors. Finance Research Letters, 10(1), 34-40.

Jones, E. R. (2004). An introduction to neural networks - A white paper. Visual Numerics, Inc., San Ramon, CA.

Klein, P. G., \& Bernanke, B. (2009). Financial regulation and supervision after the crisis: the role of the Federal Reserve. In speech at the Federal Reserve Bank of Boston $54^{\text {th }}$ Economic Conference, October (Vol. 23).

Kotkatvuori-Örnberg, J., Nikkinen, J., \& Äijö, J. (2013). Stock market correlations during the financial crisis of 2008-2009: Evidence from 50 equity markets, International Review of Financial Analysis, 28, 70-78.

Scholes, M., \& Williams, J. (1977). Estimating betas from non-synchronous data. Journal of Financial Economics, $5(3), 309-328$.

Trecroci, C. (2013). How do alphas and betas move? Uncertainty, learning and time variation in risk loadings. Oxford Bulletin of Economics and Statistics, 76(2), 257-278. 